\title{
Failure of spatial selectivity in vision
}

\author{
SUZANNE V. GATTI and HOWARD E. EGETH \\ Johns Hopkins University, Baltimore, Maryland 21218
}

\begin{abstract}
In a modified Stroop word-color paradigm, subjects were instructed to identify the color of a target patch in the center of a briefly exposed visual display and to ignore color names printed above and below the patch at distances of 1,3 , and 5 deg of visual angle. Earlier studies of selective attention to a specified location in the visual field have shown that the focus of attention subtends approximately $1 \mathrm{deg}$ of angle. That is, when distractors associated with an incompatible response are spaced within about 1 deg of the target, reaction time to the target is slower than when the distractors are not associated with an incompatible response. Our results show interference with a 5-deg separation between target and noise elements. This finding casts doubt on the utility of the notion of attentional selectivity.
\end{abstract}

Studies of visual information processing have shown that subjects are limited in their ability to focus their attention to a specific location in the visual field. In these studies (e.g., Eriksen \& Hoffman, 1972, 1974; Eriksen \& Eriksen, 1974) it has been shown that the focus of selective attention subtends approximately $1 \mathrm{deg}$ of visual angle. That is, when distractors are spaced within 1 deg of the target, subjects automatically process the distractors as well as the target. Consequently, the reaction time (RT) to process the target may be substantially slower when the distractors are associated with an incompatible response than when the distractors are associated with a compatible response, or are not associated with any specific response.

Typically, in these studies a target was presented in a specific location and the subject had to indicate which target letter (Eriksen \& Eriksen, 1974) or digit (Somers, Whitehouse, \& Egeth, Note 1) had been shown. The target element was presented either alone (no noise) or with some additional elements. These other elements might be repetitions of the target (compatible noise), an element associated with the other response (incompatible noise), or an element not associated with any overt response required in the experiment (neutral noise). For example, in the Somers et al. (Note 1) study, subjects were instructed to attend to the center of the visual display and make one response when the digit " 2 " appeared and a different response when a " 3 " appeared. The logic of these studies of selective attention to a specific location in space is that, if the subject can restrict his attention to the designated location, he should respond to the target quickly and accurately. If, however, he cannot restrict his attention, then the

This research was supported in part by a grant from the National Science Foundation to Howard E. Egeth and James R. Pomerantz (ONS 76-1227). Portions of this paper were presented at the annual meeting of the Eastern Psychological Association, Boston, April 1977. Requests for reprints may be sent to either author, Department of Psychology, Johns Hopkins University, Baltimore, Maryland 21218. noise items associated with the incompatible response would interfere with processing. Results showed that RT was much slower when, say, the digit " 2 " was flanked by " 3 "s (incompatible noise) than when the " 2 " was flanked by other "2"s (compatible noise) or "0"s (neutral noise). (The neutral noise condition serves as a control for lateral masking effects; such effects have been negligible in the reported studies.)

In Eriksen and Eriksen's (1974) study, a target letter was always presented in the center of a horizontal display. Mean RT decreased as the separation between the target and flanking noise items increased to a maximum separation of $1 \mathrm{deg}$. In that study, the interference effect at 1 deg was only about $17 \mathrm{msec}$, whereas in Eriksen and Hoffman's (1972) study an effect was obtained on the order of $70 \mathrm{msec}$ for spacings of 1 and $1.4 \mathrm{deg}$ when the target's location varied from trial to trial. In the latter study, the target and noise items were all presented on the perimeter of an imaginary circle centered on the fixation point. By keeping distance from the fovea equal for all items, noise and target items all fall on equally acute parts of the fovea. However, a bar marker or indicator had to be used to locate the target for each trial, and, thus, subjects may have encoded noise items in the course of their search for the indicator. Using a nonsearch paradigm with centered linear displays guarantees the location of the target for each trial, but confounds target-noise separation with acuity. Somers et al. (Note 1) attempted to compensate for the reduction in acuity by using relatively large numerals $(1.9 \times 1.2 \mathrm{deg})$ in the display, and did obtain strong interference effects at $2 \mathrm{deg}$. This result suggests that the size of the focus of selective attention is by no means as small as $1 \mathrm{deg}$. How large is it? In a subsequent experiment using the same sized stimuli, Gatti and Egeth (reported in Egeth, 1977) failed to obtain similar interference effects at 3 and $5 \mathrm{deg}$. When subjects were asked to report the total stimulus array in this study, they did so with perfect accuracy, even at stimulus durations of 200 msec. Thus, the lack of interference is not due 
simply to subjects being unable to see the flanking stimuli.

On the basis of these findings, we conclude that perception is partially under attentional control in that when subjects voluntarily "attend" to the location of the target, interference is only caused by stimuli within a limited distance of the target (about $2 \mathrm{deg}$ ). The obvious problem that stands in the way of easy acceptance of this conclusion is that the stimuli that caused substantial interference at 2 deg separation may not have been large enough to compensate for the reduction in acuity at 3 and 5 deg (although they were large enough to be read). Thus, a further test of the hypothesis that the spatial extent of processing is limited requires stimuli more "potent" (i.e., conspicuous) than those we have used heretofore. One possibility would be simply to make the flanking stimuli larger or brighter than before. We decided instead to take a different approach by making use of one of the strongest sources of interference available in the armamentarium of the experimental psychologist-the color-word paradigm devised by Stroop (1935).

A modified form of the Stroop word-color task was used; the target on each trial was a color patch at the center of the visual field, with either compatible or incompatible color names printed above and below the color patch at target-noise separations of 1,3 , or $5 \mathrm{deg}$ of visual angle. ${ }^{1}$ If subjects are processing fully the information in the entire display, then the incompatible color names should produce slower color namings RTs than the compatible (or no-noise) conditions, even when the noise is spaced 3 or 5 deg from the target.

\section{METHOD}

\section{Subjects}

Subjects were seven males and nine females aged 18-30 years. They were tested individually and paid $\$ 2$ for approximately $45 \mathrm{~min}$ of work. All had normal or corrected-to-normal vision.

\section{Materials and Procedure}

A Gerbrands two-channel tachistoscope was wired in circuit with a microphone and a timer to obtain vocal RTs.

A deck of 39 stimulus cards was constructed by coloring a rectangular patch $(1.2 \times 3.6 \mathrm{deg})$ in the center of each card red, blue, or green, and block printing in black ink the color names RED, BLUE, or GREEN equidistant above and below the color patch. Each letter subtended $1.2 \times 1.2 \mathrm{deg}$. For the compatible noise condition, all three items on the card were nominally the same (e.g., RED printed above and below a red color patch). For the incompatible noise condition, the printed words were nominally different from the patch (e.g., BLUE printed above and below a red color patch). For the neutral noise condition, XXXX was printed above and below the color patch to control for possible lateral masking effects. All color patches were represented with all possible combinations of color words, with the restriction that the color words printed above and below were the same (e.g., if BLUE was printed above, it would also be printed below). These three compatibility conditions were further divided into three target-noise separations of 1,3 , or $5 \mathrm{deg}$ of visual angle, measured from the edge of the patch to the edge of the color words. In addition, three cards with each of the color patches alone were constructed (no-noise condition).

Each subject was instructed to look into the tachistoscope at a centered fixation dot. After a warning signal, a stimulus card appeared and the subject was to say the name of the color patch as quickly as possible, trying not to make any errors. Stimulus duration was $200 \mathrm{msec}$, followed by the fixation dot. The deck of 39 cards was presented to each subject nine times and was reshuffled between presentations. Errors were noted as well as vocal RTs.

An instructional manipulation provided a between-subjects variable. One half of the subjects were instructed to identify the color patch in the center as quickly as possible and disregard any words written above and below (weak instructions). The remaining subjects were explicitly warned of the deleterious effect of Stroop interference and were advised that they would perform faster and more accurately if they looked only into the center, since the words above and below the target patch could impair performance (strong instructions).

\section{RESULTS AND DISCUSSION}

The first sequence of 39 cards for each subject was considered practice and was not included in the data analysis. The means of the remaining vocal RTs excluding errors are shown in Figure 1.

Figure 1 presents data for all 16 subjects collapsed over the strong and weak instruction conditions. As can be seen in the figure, subjects were considerably slower to identify the color patch when the color patch and the color names were incompatible. Further, this interference effect obtained at all three target-noise separations. Statistical analyses support these observations. An analysis of variance over all of the data for the three compatibility conditions and the three target-noise separations showed no main effect of instructions

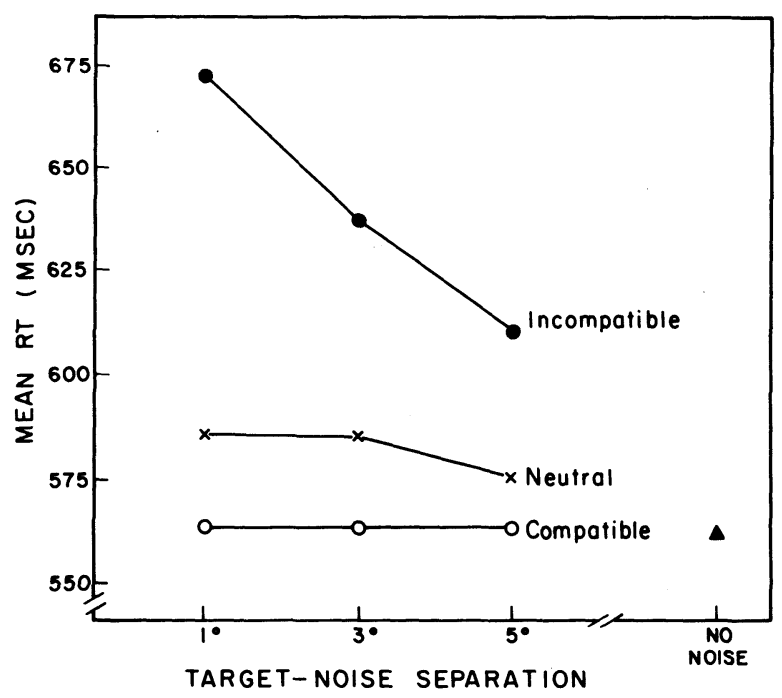

Figure 1. Mean RTs as a function of target-noise separation for the incompatible, compatible, and neutral noise conditions. Mean RT for the no-noise condition appears to the right of the figure. 
$[F(1,14)<1]$ and no interactions of the instruction factor with any other factor. However, the main effects of separation $[\mathrm{F}(2,28)=9.24, \mathrm{p}<.001]$ and compatibility $[\mathrm{F}(2,28=43.24, \mathrm{p}<.001]$ were reliable, as was their interaction $[\mathrm{F}(4,56)=13.45, \mathrm{p}<.001]$. To see if there was significant interference at the greatest separation, a $t$ test for correlated measures between the means of the incompatible and neutral conditions at $5 \mathrm{deg}$ was performed. The interference effect proved highly reliable $[t(15)=5.25, p<.001$ two-tailed $]$, showing that the irrelevant color words interfered with target processing even when the words were spaced 5 deg from the target. Although interference decreases with increasing separation, this does not necessarily mean that there is a falloff of attention at these larger separations; it may be more simply interpreted as a fall-off in acuity as the color words are moved out into the periphery.

The finding that Stroop interference obtained over a distance of 5 deg seems to have important implications for theories of selective attention. Specifically, we employed a task known to increase RTs due to subjects' mandatory processing of conflicting information (Dyer, 1973a, 1973b). Whether this increase in processing time is due to input or response factors is not at issue here, but rather that subjects attend to the irrelevant information at all. Even when subjects were warned of the Stroop effect, they nevertheless performed poorly when the stimulus information conflicted, as shown in the strong instructions condition. Eriksen and Eriksen (1974) and Eriksen and Hoffman (1972) suggested that noise information within about $1 \mathrm{deg}$ of the target is automatically processed, whereas information spaced outside of the 1-deg focus may or may not be processed, depending on the amount of energy or capacity left over after processing the information in the focus. Eriksen does allow for some slight, asymptotic interference effects at separations greater than approximately $1 \mathrm{deg}$; "Once the noise is removed beyond a degree of an angle, how much further away it is does not appear to make any difference" (personal communication; see also Eriksen \& Eriksen, 1974, p. 144). However, in our study, the interference at $5 \mathrm{deg}$ was still considerable, approximately $35 \mathrm{msec}$. Any attenuation of the interference at the larger separations may simply be attributed to reduced acuity rather than to attenuation of attention.

Also, according to Eriksen's model, when capacity reserves allow processing of information outside of the focus, the extent of processing in the outlying fringe area is only to an incomplete, gross level of analysis. In our experiment, the word names should not have interfered at the larger separations unless we assume either that the focus may be larger than $1 \mathrm{deg}$, to allow for the higher level of processing for word names (at least through the identification stage), or that processing depth in the fringe area is more elaborate than to just the gross level that has been proposed. Alternately, it may be more parsimonious to adopt a model of attention where all of the input (within the usual physiological constraints such as acuity) is identified and then selected for task relevancy (e.g., Deutsch \& Deutsch, 1963; Shiffrin \& Grantham, 1974). In a recent paper, Keren, O'Hara, and Skelton (in press) showed that noise elements may be processed to different levels depending on the "depth" of similarity between the codes of the target and noise items. In our study, the noise items (word names) were only irrelevant in the sense of spatial location. However, the color-word names may have been so relevant or meaningful to the task that the subjects were unable to gate out the information.

Our chief finding is that subjects cannot focus attention sufficiently to gate out information from as far as $5 \mathrm{deg}$ from the centrally located target. Thus, the concept of a strictly limited size of the attentional focus, or even the concept of a focus at all, may not be a valid one, since selectivity may depend on more cognitive factors, such as the meaningfulness of the noise items and the subjects' ability to successfully ignore "irrelevant" information.

Our data are consistent with the idea that perceptual processing is not under attentional control, but they certainly do not prove the case. The problem is that we cannot conclude from our data that subjects do not filter (i.e., attenuate) irrelevant information at all. It may be that our subjects were filtering the noise to some extent and that the interference effects we observed were smaller than would have been obtained if subjects had not been able to attend selectively. It is important to note that this argument applies with equal force to other studies in which "unattended" stimuli are shown to capture attention or otherwise disrupt processing of attended material. Such demonstrations abound in the auditory literature (e.g., Corteen \& Wood, 1972; Lewis, 1970; Moray, 1959). Along with our study and the earlier work of Eriksen and his colleagues, these studies show that selectivity is not perfect; they do not show that it is nonexistent.

\section{REFERENCE NOTE}

1. Somers, P., Whitehouse, P., \& Egeth, H. E. Failure of attentional selectivity in a nonsearch detection task (Tech. Dep. 87). Baltimore: Johns Hopkins University, Department of Psychology, August 1976.

\section{REFERENCES}

Corteen, R. S., \& Wood, B. Autonomic responses to shockassociated words in an unattended channel. Journal of Experimental Psychology, 1972, 94, 308-313.

Deutsch, J. A., \& Deutsch, D. Attention: Some theoretical considerations. Psychological Review, 1963, 70, 80-90.

DYER, F. N. The Stroop phenomenon and its use in the study of perceptual, cognitive and response processes. Memory \& Cognition, 1973, 1, 106-110. (a)

DYER, F. N. Interference and facilitation for color naming with separate bilateral presentations of the word and color. Journal of Experimental Psychology, 1973, 99, 314-317. (b) 
Egeth, H. E. Attention and preattention. In G. H. Bower (Ed.), The psychology of learring and motivation (Vol. 11). New York: Academic Press, 1977.

Eriksen, B. A., \& ERIKSEN, C. W. Effects of noise letters upon the identification of a target letter in a nonsearch task. Perception \& Psychophysics, 1974, 16, 143-149.

Eriksen, C. W., \& Hoffman, J. E. Temporal and spatial characteristics of selective encoding from visual displays. Perception \& Psychophysics, 1972, 12, 201-204.

Eriksen, C. W., \& Hoffman, J. E. Selective attention: Noise suppression or signal enhancement? Bulletin of the Psychonomic Society, 1974, 4, 587-589.

Keren, G., O'Hara, W. P., \& Skelton, J. M. Levels of processing and attentional control. Journal of Experimental Psychology: Human Perception and Performance, in press.

LEwIs, J. L. Semantic processing of unattended messages using dichotic listening. Journal of Experimental Psychology, 1970, 85, 225-228.

Moray, N. Attention in dichotic listening: Affective cues and the influence of instructions. Quarterly Journal of Experimental Psychology, 1959, 11, 56-60.
Shiffrin, R. M., \& Grantham, D. W. Can attention be allocated to sensory modalities? Perception \& Psychophysics, 1974, 15 460-474.

STROop, J. R. Studies of interference in serial verbal reactions. Journal of Experimental Psychology, 1935, 18, 643-662.

\section{NOTE}

1. A superficially similar experiment was performed by Dyer (1973b), who showed interference effects at 4 deg separation between the color and interfering word name. However, in Dyer's study the subjects never knew from one trial to the next the relative locations of the color and word with respect to the fixation points. Thus, it would not have been appropriate for subjects to try to limit their focus to a specific location in the visual field.

(Received for publication November 15, 1977.) 